\title{
Hepatic abscess as a complication of an abscessed gastric lipoma
}

\author{
Maria Jesús Gayán Belmonte, Elena Parlorio de Andrés, Carmen María Botía González
}

Radiology Department, Hospital Universitario Morales Meseguer, Murcia, Spain

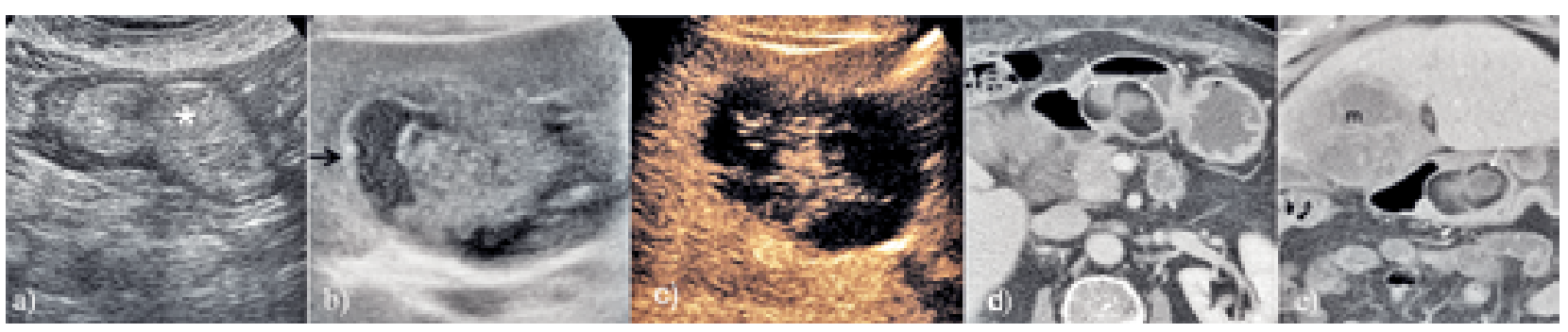

Fig 1. a) and b) Ultrasound images show well-defined lobulated submucosal mass in the gastric antrum (white asterisk in a) and a rounded predominantly liquid with internal echogenic areas mass in the liver (black arrow in b); c) CEUS images of the liver lesion with a "honeycomb pattern"; axial (d) and coronal projection (e) of contrast-enhanced multiphasic multidetector computer tomography images evidence the submucosal mass in the gastric antrum with liquid collections inside (whitearrows) and the hepatic mass (m).

\section{To the Editor,}

A 70-year-old female attended to our hospital complaining of a pain in the upper abdomen for the last two months that had worsened in the last 72 hours. The pain was accompanied with bilious vomits, chills and fever $\left(38.9^{\circ} \mathrm{C}\right)$. The laboratory tests performed were normal except for the presence of leukocytosis $\left(16,000 / \mathrm{mm}^{3}\right)$.

The abdominal ultrasound (US) showed a well-defined lobulated hyperechoic with hypoechoic foci submucosal mass in the posterior wall of the gastric antrum that measured $6.5 \mathrm{~cm} \times 2.5 \mathrm{~cm}$ (fig $1 \mathrm{a}$ ), but to our surprise, on US we also found a large $(7 \mathrm{~cm})$ rounded, predominantly liquid with internal echogenic areas, mass in the left lobe of the liver (fig $1 \mathrm{~b}$ ). The liver lesion evidenced a

Received 01.07.2017 Accepted 12.07.2017

Med Ultrason

2017, Vol. 19, No 3, 336-337, DOI: 10.11152/mu-1171

Corresponding author: Maria Jesús Gayán Belmonte

Radiology Department, Hospital Universitario

Morales Meseguer.

Calle Marqués de los Vélez, s/n 30008

Murcia, Spain

Phone: +0034 968360900

E-mail: mariajesus.gayan@gmail.com "honeycomb pattern" on Contrast-Enhanced US (CEUS), with non-enhancing necrotic areas and enhancing internal septae (fig1c).

On Multiphasic Multidetector computer tomography (CT) the submucosal mass in the gastric antrum had fat density and liquid collections inside (fig 1d,e). The hepatic mass was heterogeneous but predominantly hypodense and had a perilesional hypodense area which showed progressive enhancement in the following phases due to hyperemic inflammatory effects on the adjacent liver.

Subsequently, the patient was referred to the Digestive Department, where an endoscopy revealed a submucosal mass in the posterolateral wall of the gastric antrum with an ulcerated area on it surface. Finally, the pathological study of the gastric mass, which was surgically resected, revealed an abscessed submucosal gastric lipo$\mathrm{ma}$, and the microbiological study of the hepatic abscess, which was radiologically drainaged, revealed Gemella morbillorum.

Gastric lipomas comprise 2-3\% of the gastric benign tumors and are composed of mature fat cells surrounded by a fibrous capsule $[1,2]$. They are usually located in the gastric antrum, and present as solitary intramural le- 
sions incidentally detected in asymtomatic patients [2] that may, however, cause ulceration, gastrointestinal bleeding or intermittent gastric obstruction if they are large [1]. CT is the imaging modality of choice for the study of these tumors, the tumors appearing as solitary, well-defined submucosal lesions with homogeneous fat attenuation [2].

Hepatic abscesses are common in daily practice. Pyogenic liver abscesses usually appear in patients with predisposing conditions such as diabetes, hepatobiliary malignancy or immunosuppression [3]. They typically present as hypoechoic lesions with irregular thickened walls and internal septae on US with "honey-comb" appearance on CEUS [3].
There are very few references regarding pyogenic liver abscesses as a complication of gastric tumors, and all of them concern Gastrointestinal Stromal Tumors (GISTs).

\section{References}

1. Kang HC, Menias CO, Gaballah AH, et al. Beyond the GIST: mesenchymal tumors of the stomach. Radiographics 2013;33:1673-1690.

2. Virmani V, Khandelwal A, Sethi V, Fraser-Hill M, Fasih N, Kielar A. Neoplastic stomach lesions and their mimickers: spectrum of imaging manifestations. Cancer Imaging 2012;12:269-278.

3. Chaubal N, Joshi M, Bam A, Chaubal R. Contrast enhanced ultrasound of focal liver lesions. Semin Roentgenol 2016;51:334-357.

\title{
Adductor muscle tear? Ultrasonographic imaging of avulsion injury of the iliopsoas tendon
}

\author{
Ke-Vin Chang, Wei-Ting Wu, Der-Sheng Han
}

Department of Physical Medicine and Rehabilitation, National Taiwan University Hospital, Bei-Hu Branch and National Taiwan University College of Medicine, Taipei, Taiwan

\section{To the Editor}

A 12-year-old boy complained of left medial thigh pain after a fall while he attempted to play volleyball. The pain prevented him from running and was not responsive to topical analgesics. He visited a sports clinic, where adductor muscle injury was first impressed with a negative finding on radiography. An oral non-steroid anti-inflammatory drug was prescribed but his pain persisted despite 2 weeks of medication. He was referred for an ultrasonographic examination under the suspicion of

Received 03.06.2017 Accepted 26.06.2017

Med Ultrason

2017, Vol. 19, No 3, 337-338, DOI: 10.11152/mu-1145

Corresponding author: Ke-Vin Chang, $\mathrm{MD}, \mathrm{PhD}$

Department of Physical Medicine and

Rehabilitation, National Taiwan University

Hospital, Bei-Hu Branch and National Taiwan

University College of Medicine, Taipei, Taiwan

E-mail: kvchang011@gmail.com adductor muscle tears. The transducer was first placed over the proximal medial thigh where the adductor muscle group appeared normal. Although no effusion was observed inside the anterior hip recess, the patient felt intense pain while the transducer was glided over the distal edge of the femoral neck. He was repositioned by abducting and externally rotating the hip to allow placement of the transducer on the posterior medial hip. A bony chip was noticed at the insertion of the iliopsoas tendon, corresponding to the tip of the lesser trochanter (fig 1, Video 1 , active in the journal site - http://www.medultrason. ro). Iliopsoas tendon avulsion injury was diagnosed and subsequent physical therapy that targeted the involved region was administered. He reported relief of the pain after treatment for 1 month.

The iliopsoas muscle is a combination of the psoas major and iliacus muscles, and inserts on the lesser trochanter of the femur [1]. The muscle is a strong hip flexor and is vulnerable to hyperextension injury. A pathological 


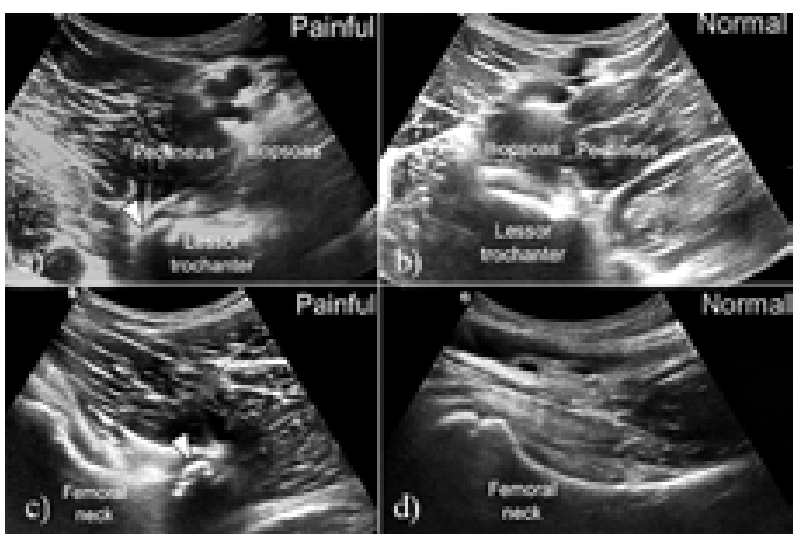

Fig 1. Ultrasonography image of the iliopsoas tendon attachment in the short - (A and B) and long-axis views $(\mathrm{C}$ and $\mathrm{D})$ on the painful and normal sides. Arrowhead: avulsed fragment.

iliopsoas myotendinous complex uncommonly occurs in the general population but is more prevalent in athletes and dancers. The pertinent symptoms include groin pain and limitation of hip movement, and can occur in patients with hip arthropathy or pathology affecting the adductor, pectineus, and rectus femoris muscles. Teenagers are at a higher risk of apophyseal injuries such as the avulsion of the iliopsoas tendon in our case.

Ultrasonography has emerged as a valid tool to differentiate anterior hip disorder, especially in patients with sport injuries [1]. Anterior hip recess effusion, iliopsoas bursitis, avulsion of the direct tendon of the rectus femoris, and tears of the adductor muscle are common etiologies identified on ultrasonography [2]. There is a paucity of literature reporting ultrasonographic imaging of apophyseal injury of the iliopsoas tendon. Examination of the iliopsoas tendon attachment requires full exposure of the posterior medial aspect of the thigh by abducting and externally rotating the hip [3]. Its attachment, the lesser trochanter, can be scanned in the axial plane of the femur and appears as an elongated spine extending medially from the distal portion of the femoral neck. The medial femoral circumflex artery, which courses underneath the

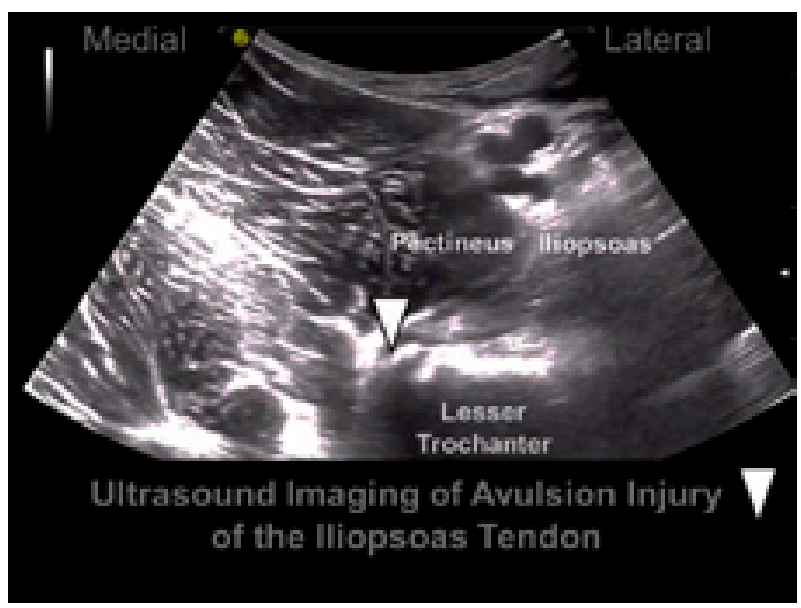

Video 1.

iliopsoas tendon, should be examined for possible collateral damage. The present case highlights the importance of examining the attachment of the iliopsoas tendon in teenagers with sport injuries and the requirement of specific positioning to visualize the lesser trochanter by using ultrasonography.

Acknowledgment: The current research is supported by National Taiwan University Hospital, Bei-Hu branch and Taiwan Society of Ultrasound in Medicine

\section{Reference}

1. Ozcakar L, Kara M, Chang KV, et al. EURO-MUSCULUS/ USPRM. Basic scanning protocols for hip. Eur J Phys Rehabil Med 2015;51:635-640.

2. Hung CY, Chang KV, Ozcakar L. Avascular Necrosis of the Femoral Head Masquerading as Iliopsoas Bursitis: Imaging With Ultrasound and Magnetic Resonance. Am J Phys Med Rehabil 2016;95:e24-e25.

3. Balius R, Pedret C, Blasi M, et al. Sonographic evaluation of the distal iliopsoas tendon using a new approach. J Ultrasound Med 2014;33:2021-2030. 


\title{
Ultrasonographic diagnosis of heterotopic ossification and secondary nerve entrapments in a patient with spinal cord injury
}

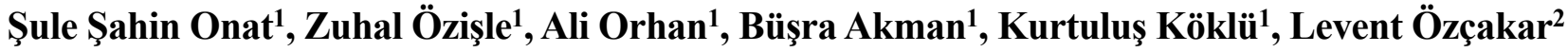

${ }^{1}$ Ankara Physical and Rehabilitation Medicine Training and Research Center, ${ }^{2}$ Hacettepe University Medical School Department of Physical and Rehabilitation Medicine, Ankara, Turkey

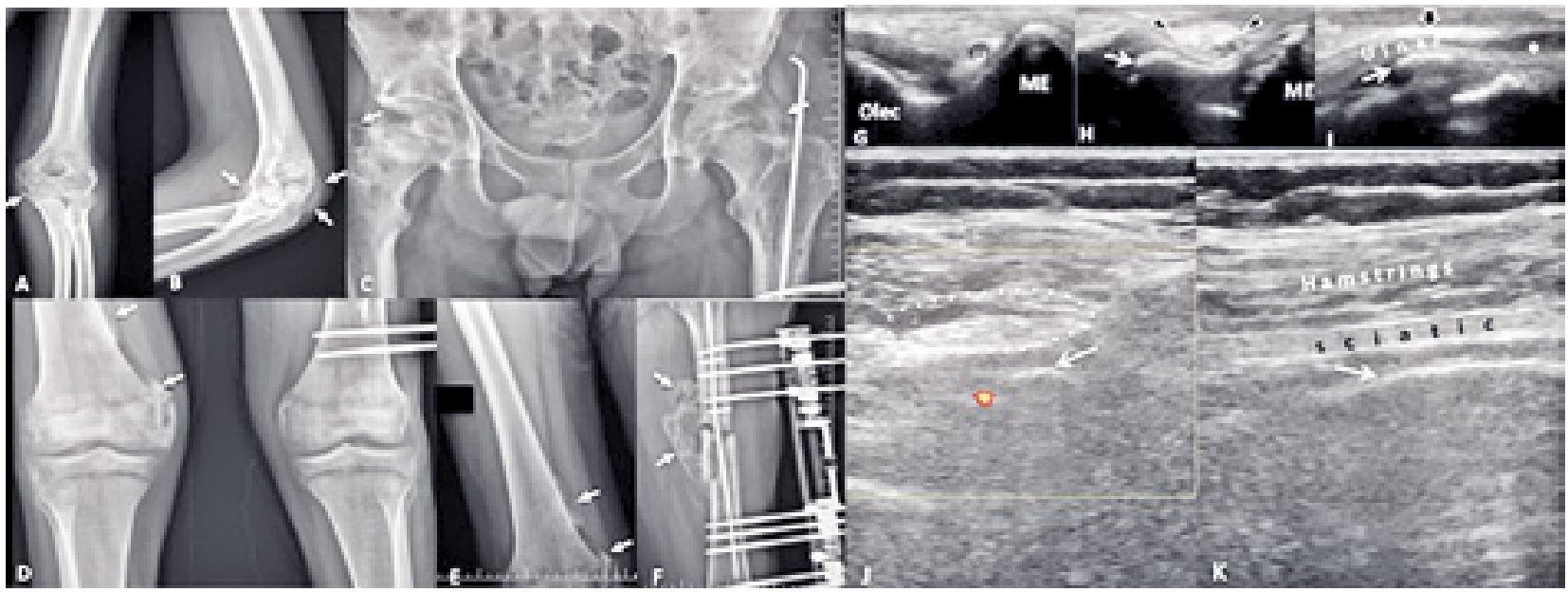

Fig 1. Antero-posterior and lateral radiographs show heterotopic ossification (white arrows) around the elbow (A, B), bilateral hip joints $(C)$, and the right knee (D, E) and callus formation at the proximal femur (F). Ultrasound imaging (axial view) shows the right normal ulnar nerve $(\mathrm{u})(\mathrm{G})$ and the left ulnar nerve (black arrowheads) as swollen with hypoechoic, edematous and enlarged (asterisk) just proximal to the cubital tunnel (H). In longitudinal view (I) note the compression of the nerve (black arrow) due to the heterotopic ossification (white arrows). Posterior axial $(\mathrm{J})$ and longitudinal $(\mathrm{K})$ scans of the proximal thigh illustrate the close relationship between the heterotopic ossification (white arrows) and the sciatic nerve (white dashed line). Olec: Olecranon, ME: medial epicondyle

\section{To the Editor,}

A 24-year-old man (with a previous history of traumatic L2 vertebra burst, left fragmented femur, and distal radius fractures one year ago) was admitted for rehabilitation. He had significant limitations in the left elbow,

Received 04.06.2017 Accepted 12.06.2017

Med Ultrason

2017, Vol. 19, No 3, 339-340, DOI: 10.11152/mu-1121

Corresponding author: Şule Şahin Onat

Ankara Physical and Rehabilitation Medicine

Training and Research Center

3 Türkocağı street, Sthhiye 06230, Ankara, Turkey

Phone: +9005053136848, Fax:+903123103230

E-mail: sahinsulester@gmail.com bilateral hip, and knee joint motions, hypoesthesia on the medial side of the left forearm, 4th and 5th fingers (palmar side) and below the right L2 and left L3 dermatomes. Left finger abduction was $3 / 5$, bilateral hip flexion was $4+/ 5$, hip extension (right-left) was $3-2 / 5$, ankle dorsiflexion (right-left) was $3-1 / 5$ in muscle strength test. He had interosseous atrophies, positive Froment's and Wartenberg's signs. He had voluntary anal contractions and positive bulbocavernose reflex.

Laboratory tests were normal except alkaline phosphatase level of $167 \mathrm{u} / \mathrm{L}(\mathrm{N}: 38-155 \mathrm{u} / \mathrm{L})$. Radiographs demonstrated heterotopic ossification (HO) around the left elbow, bilateral hip, right knee joints, and left femur (fig 1A-F). Electrodiagnostic evaluations revealed 
left ulnar nerve injury around the elbow. Sciatic nerve could not be well assessed due to the external fixator and the loss of muscle mass. Ultrasound (US) imaging was performed and clearly showed the entrapment of the sciatic and ulnar nerves (fig 1G-K). Overall, the patient was diagnosed with spinal cord injury (L2 level, ASIA D) and concomitant sciatic and ulnar nerve injuries due to $\mathrm{HO}$.

$\mathrm{HO}$ is defined as the formation of trabecular bone outside the normal sites of the skeletal structure i.e. materializing in soft tissues in places where it does not usually exist [1]. Although its etiology remains unclear, clinical risk factors for $\mathrm{HO}$ include trauma, amputation, traumatic brain injury, spinal cord injury, thermal injury, major hip arthroplasty, and other major orthopedic surgeries [1]. The aberrant bony growth is generally diagnosed by radiography, ultrasonography, three-phase bone scanning, computed tomography (CT) and magnetic resonance imaging (MRI) [2]. Three-phase bone scanning is very sensitive for detecting early $\mathrm{HO}$; however, it has low specificity. Radiography, CT and MRI are either expensive or have the risk of radiation exposure. As such, US appears to be the most suitable first-line imaging modality for the diagnosis of $\mathrm{HO}[2,3]$. It can show $\mathrm{HO}$ in the early period (even when x-rays are negative) with characteristic findings in B-mode (i.e. zone phenomenon, cloudy appearance) and power Doppler imaging (i.e. peripheral hypervascularization) [4]. Moreover, as in our case, any soft tissue injury due to HO (e.g. nerve or vascular compression) can readily/substantially be visualized with US. For nerves, entrapment findings would include fusiform enlargement proximal to the site of compression, change in shape (swelling), decreased echogenicity and intraneural edema [5].

The nerve injuries due to $\mathrm{HO}$ are quite rare [6-8]; in this report, we aimed to imply that US can be a trustwor- thy initial imaging tool for prompt/early diagnosis of HO and the accompanying nerve entrapments, especially in patients in whom other imaging modality cannot be easily/effectively performed due to various medical conditions (spinal cord injury, external fixator, etc.)

\section{References}

1. Akkaya N. Ultrasound imaging in rehabilitation settings. In: Özçakar L, De Myunk Martine, (eds). Musculoskeletal Ultrasound, 1st Edition, Milan, Italy, Edi-Ermes, 2014:185197.

2. Ekiz T, Yıldızgören MT, Yetişgin A. Musculoskeletal ultrasonography bypasses the diagnostic and radiological challenges in heterotopic ossification. Singapore Med J 2014;55:604.

3. Özçakar L, Kara M, Chang KV, et al. Nineteen reasons why physiatrists should do musculoskeletal ultrasound: EURO-MUSCULUS/USPRM recommendations. Am J Phys Med Rehabil 2015;94:e45-e49.

4. Ozçakar L, Carli AB, Tok F, Tekin L, Akkaya N, Kara M. The utility of musculoskeletal ultrasound in rehabilitation settings. Am J Phys Med Rehabil 2013;92:805-817.

5. Kara M, Özçakar L, De Muynck M, Tok F, Vanderstraeten G. Musculoskeletal ultrasound for peripheral nerve lesions. Eur J Phys Rehabil Med 2012;48:665-674.

6. Salga M, Jourdan C, Durand MC, et al. Sciatic nerve compression by neurogenic heterotopic ossification: use of CT to determine surgical indications. Skeletal Radiol 2015;44:233-240.

7. Kara M, Ekiz T, Öztürk GT, Onat ŞŞ, Özçakar L. Heterotopic ossification and peripheral nerve entrapment: Ultrasound is a Must-use imaging modality. Pain Med 2015;16:1643-1644.

8. Kara M, Yalçın S, Yenigün D, Tiftik T, Malas FÜ, Özçakar L. Heterotopic ossification and cubital tunnel syndrome in traumatic brain injury: Ultrasound 'sees' both. J Back Musculoskelet Rehabil 2015;28:415-417. 